\title{
Further Improvements in the Understanding of Isotropic Loop Quantum Cosmology
}

\author{
M. Martín-Benito $*$ G. A. Mena Marugán $]^{\dagger}$ and J. Olmedd \\ Instituto de Estructura de la Materia, CSIC, Serrano 121, 28006 Madrid, Spain
}

The flat, homogeneous, and isotropic universe with a massless scalar field is a paradigmatic model in Loop Quantum Cosmology. In spite of the prominent role that the model has played in the development of this branch of physics, there still remain some aspects of its quantization which deserve a more detailed discussion. These aspects include the kinematical resolution of the cosmological singularity, the precise relation between the solutions of the densitized and non-densitized versions of the quantum Hamiltonian constraint, the possibility of identifying superselection sectors which are as simple as possible, and a clear comprehension of the Wheeler-DeWitt (WDW) limit associated with the theory in those sectors. We propose an alternative operator to represent the Hamiltonian constraint which is specially suitable to deal with all these issues in a detailed and satisfactory way. In particular, with our constraint operator, the singularity decouples in the kinematical Hilbert space and can be removed already at this level. Thanks to this fact, we can densitize the quantum Hamiltonian constraint in a well-controlled manner. Besides, together with the physical observables, this constraint superselects simple sectors for the universe volume, with a discrete support contained in a single semiaxis of the real line and for which the basic functions that encode the information about the geometry possess optimal physical properties. Namely, they provide a no-boundary description around the cosmological singularity and admit a well-defined WDW limit in terms of standing waves. Both properties explain the presence of a generic quantum bounce replacing the classical singularity at a fundamental level, in contrast with previous studies where the bounce was proved in concrete regimes -focusing on states with a marked semiclassical behavior- or for a simplified model.

PACS numbers: 04.60.Pp,04.60.Kz,98.80.Qc

\footnotetext{
* merce.martin@iem.cfmac.csic.es

$\dagger$ mena@iem.cfmac.csic.es

‡ olmedo@iem.cfmac.csic.es
} 


\section{INTRODUCTION}

Loop Quantum Gravity (LQG) [1 $\sqrt[3]{3}]$ is one of the most promising candidates for a theory of quantum gravity. Nonetheless, although this quantization program of General Relativity has been extensively developed, it has not been completed yet. In order to test the program and obtain physical predictions in simple situations of interest, Loop Quantum Cosmology (LQC) implements the quantization procedures of LQG in symmetry reduced models [4]. This field has suffered a major breakthrough in recent years thanks to the large number of studies carried out in models such as flat FRW cosmologies [5-11], FRW universes with other topologies [12 14] or with a non-vanishing cosmological constant [15], anisotropic Bianchi I models [16 19], or even some inhomogeneous scenarios [20].

In this work, we will focus our attention on the flat FRW model with a massless scalar field, which is the most prominent system studied so far in LQC. In fact, even though this is the simplest cosmological model with non-trivial dynamics, its polymeric quantization has already provided relevant results [5 7], such as the validity of the classical dynamics for semiclassical states in LQC in the region far away from the classical singularity and, more importantly, the replacement of this big bang singularity by a quantum bounce. In addition, the robustness of these results has been carefully checked [8, 9]. Besides, those studies, together with other complementary analyses [10, 11], have served to rigorously establish the mathematical foundations of LQC.

Nevertheless, despite the extensive analysis of this model performed up to date, there are still some issues which are not completely clear and need a more careful discussion in order to have a thorough understanding of the theory. In particular, the points that we have in mind involve, on the one hand, a rigorous densitization of the Hamiltonian constraint (with respect to the volume of the universe) at the quantum level -a step which is carried out in order to obtain a densitized Hamiltonian constraint that is easier to solve than the non-densitized one arising naturally from LQG-, and, on the other hand, the superselection of the kinematical Hilbert space in different sectors which are intended to be as simple as possible while possessing optimal physical properties. Among such properties, we are interested in the existence of a regime with a well-defined WDW limit and where general features of the states provide a basis to explain the occurrence of a quantum bounce.

With the aim of investigating these questions while keeping a rigorous mathematical control of all the steps in the discussion, we will adopt a new prescription when promoting the Hamiltonian constraint of the cosmological model to a symmetric operator. Actually, our quantum Hamiltonian constraint results more suitable than previous proposals inasmuch as it clarifies the issues stated above. One of the noticeable features of our prescription is the resolution of the cosmological singularity already at the kinematical level, in the sense that our Hamiltonian constraint allows us to decouple the zero-volume state from the rest of states in the kinematical Hilbert space, so that the kernel of the volume operator can be removed from the quantum theory (in a certain sense, this implements ideas from Bojowald 21]). Once the singularity has been eliminated, we are able to formally establish a bijection between the solutions of the densitized and non-densitized versions of the quantum Hamiltonian constraint, an aspect which was not properly considered in previous analyses. Another important feature of our Hamiltonian constraint is that it decouples states with opposite orientations of the triad. Owing to this fact, we can identify in our theory superselection sectors which are simpler than those obtained in Refs. [7, 8], since they are supported in discrete semilattices contained in a single semiaxis of the real line, instead of the whole line. Therefore, in our theory we can restrict the study to (e.g.) the positive semiaxis in a natural way, without the need to introduce any particular boundary condition or appeal to the parity symmetry discussed in Ref. 7] (this symmetry is straightforward to impose in our case). The simplicity of our superselection sectors allows us to gain physical intuition and improve our knowledge about the physical consequences of the loop quantization. On the one hand, the basic elements which encode the information about the geometry, namely the generalized eigenfunctions of the gravitational part of the constraint (defined as a self-adjoint operator), are each explicitly determined by a single piece of initial data. They "arise" in a single section of minimum non-zero volume, without crossing the singularity to the sector of opposite triad orientation and without satisfying any kind of boundary condition. In this sense, the self-adjointness of the gravitational part of the constraint leads to a no-boundary description. On the other hand, whereas this part of the constraint is an operator with non-degenerate spectrum, its analog in the WDW theory is two-fold degenerate. This property, together with the fact that it is a real difference operator in the volume representation, implies that its eigenfunctions have a WDW limit with

the form of an exact standing wave, i.e., each eigenfunction converges to a specific linear combination of the two analog WDW elements (one of them contracting in volume and the other one expanding) where they both contribute with equal amplitudes. Since, owing to the no-boundary property, the outgoing flux 
associated with the expanding component must become incoming flux corresponding to the contracting component, and vice versa, a quantum bounce must happen. In this way, we will be able to explain the existence of a quantum bounce, showing that it is a direct consequence of the quantum geometry underlying LQC and hence a fundamental feature of the theory.

The paper is organized as follows. In Sec. III we review the classical framework and the quantum kinematics. We choose a new, suitable symmetric ordering for the Hamiltonian constraint and densitize it in Sec. III. The gravitational part of the constraint is analyzed in detail in Sec. IV. We then impose the constraint in Sec. V] obtaining the physical Hilbert space. In Sec. VI we compare different aspects of our proposal and of previous ones, showing the advantages of our approach. Finally, in Sec. VII we discuss the main conclusions of our analysis.

\section{THE FLAT, HOMOGENEOUS, AND ISOTROPIC MODEL}

We briefly summarize here the classical formulation of the model and its kinematical treatment in LQC. For more details, see Refs. [5, 7].

\section{A. The Classical Approach}

In the flat model under study, the spatial hypersurfaces are non-compact. Therefore, in principle, the spatial integrals arising in this system diverge. In order to avoid this problem, one introduces a Euclidean fiducial 3-metric and restrict the integrals to a given cell, which is cubic with respect to the fiducial metric and whose fiducial volume is $V_{0}[5]$. Owing to the imposition of homogeneity, the spatial diffeomorphism freedom is fixed and the associated constraints are trivially satisfied. Furthermore, the internal [SU(2)] gauge freedom is removed by choosing a diagonal gauge, in which the connection and the densitized triad are respectively given by

$$
A_{a}^{i}=c V_{0}^{-1 / 3} \delta_{a}^{i} \text { and } E_{i}^{a}=p V_{0}^{-2 / 3} \delta_{i}^{a} .
$$

So, one can identify the internal indices $i=1,2,3$ with those for the tangent space $a=1,2,3$, and the three directions are equivalent owing to the isotropy. In this way, the two quantities $c$ and $p$ represent the only degrees of freedom of the geometry. This choice of parameterization for $A_{a}^{i}$ and $E_{i}^{a}$ leaves all the relevant physical quantities independent of the fiducial structures, as it is the case for the symplectic structure, determined by the Poisson brackets $\{c, p\}=8 \pi G \gamma / 3$, where $G$ is the Newton constant and $\gamma>0$ is the Immirzi parameter.

In order to have non-trivial dynamics, it is necessary to add matter degrees of freedom. The simplest possibility is a homogeneous massless scalar field $\phi$, with conjugate momentum $p_{\phi}$, such that $\left\{\phi, p_{\phi}\right\}=1$.

The model is then subject only to the Hamiltonian constraint. Making use of the spatial homogeneity, the integrated form of this constraint for any lapse function $N$ is $\mathbb{C}(N)=N C$, where the Hamiltonian constraint

$$
C=-\frac{6}{\gamma^{2}} c^{2} \sqrt{|p|}+8 \pi G \frac{p_{\phi}^{2}}{|p|^{3 / 2}}
$$

has the standard densitization used in LQG. It is easy to check that, classically, both $p$ and $\phi$ are monotonous functions of the proper time. Usually, $\phi$ is regarded as an internal time, with all quantities evolving as functions of it. Then, the classical solutions represent expanding or contracting universes, and all of them are singular.

\section{B. Quantum Representation}

In LQG, the gauge invariant information about the phase space is captured in holonomies of $\operatorname{su}(2)$ connections and fluxes through surfaces. Similarly, in LQC one adopts as basic variables holonomies along straight edges of oriented coordinate length $\mu V_{0}^{1 / 3}$ in the fiducial directions -that are given by $h_{i}^{\mu}(c)=e^{\mu c \tau_{i}}$ for the direction $i[22]$ - and fluxes through squares normal to these fiducial directions -which are equal to $p$ up to unimportant factors. The configuration algebra $\mathrm{Cyl}_{S}$ is the algebra of almost 
periodic functions of $c$ 23, 24] generated by the matrix elements of the holonomies, $\mathcal{N}_{\mu}=e^{i \mu c / 2}$. In the momentum representation, the states defined by these matrix elements are denoted by $|\mu\rangle$. The Cauchy completion of $\mathrm{Cyl}_{S}$ with respect to the discrete norm $\left\langle\mu \mid \mu^{\prime}\right\rangle=\delta_{\mu \mu^{\prime}}$ provides the gravitational part of the kinematical Hilbert space $\mathcal{H}_{\text {kin }}^{\text {grav }}$. The operator $\hat{p}$ has a diagonal action on the basis formed by the states $|\mu\rangle$, whereas $\hat{\mathcal{N}}_{\mu}$ shifts the state $\left|\mu^{\prime}\right\rangle$ to $\left|\mu^{\prime}+\mu\right\rangle$.

The standard procedure in LQC to define the curvature of the su(2)-connection is to express it in terms of holonomies along closed loops and shrink their area to the minimum non-zero value $\Delta$ allowed by LQG, which is proportional to the Immirzi parameter and to the square of the Planck length $l_{\mathrm{Pl}}=\sqrt{G \hbar}$. Then, the condition that the physical area of a square with sides of (minimum) fiducial length $\bar{\mu} V_{0}^{1 / 3}$ equals $\Delta$ 7] leads to the operator relation

$$
\frac{\widehat{1}}{\bar{\mu}}=\frac{\widehat{\sqrt{|p|}}}{\sqrt{\Delta}}
$$

Since the corresponding shift produced by $\hat{\mathcal{N}}_{\bar{\mu}}$ is not constant in the basis of states $|\mu\rangle$, it is convenient to relabel these states by introducing an affine parameter $v(\mu)$, which is proportional to the respective eigenvalue of the physical volume operator. In this manner, $\hat{\mathcal{N}}_{\bar{\mu}}$ produces a constant increment in the volume, $\hat{\mathcal{N}}_{\bar{\mu}}|v\rangle=|v+1\rangle$, while $\hat{p}|v\rangle=\operatorname{sign}(v)\left(2 \pi \gamma l_{\mathrm{Pl}}^{2} \sqrt{\Delta}|v|\right)^{2 / 3}|v\rangle$.

On the other hand, for the matter part of the kinematical Hilbert space one takes the standard representation space of square integrable functions with respect to the Lebesgue measure, namely $\mathcal{H}_{\text {kin }}^{\text {mat }}=$ $L^{2}(\mathbb{R}, d \phi)$. In total, the kinematical Hilbert space is $\mathcal{H}_{\text {kin }}=\mathcal{H}_{\text {kin }}^{\text {grav }} \otimes \mathcal{H}_{\text {kin }}^{\text {mat }}$.

\section{THE HAMILTONIAN CONSTRAINT OPERATOR}

Once the kinematical structure of the quantization has been introduced, we can proceed to represent the Hamiltonian constraint $C$ as an operator. We will choose a symmetric ordering for the constraint which differs from the orderings considered in Refs. [7, 8] but, as we will see, results more suitable.

As usual in LQC, to represent the factor $|p|^{-3 / 2}$ in the second term of Eq. (2) one appeals to Thiemann's procedure [1], rewriting it in terms of Poisson brackets of holonomies with the physical volume $V:=|p|^{3 / 2}$. On the other hand, in the gravitational part of the constraint, one expresses the curvature of the su(2)connection using holonomies as explained above, in order to obtain a well defined operator [5 7 ].

In earlier works, the quantum Hamiltonian constraint was constructed along these lines, adopting certain choices of factor ordering directly for the isotropic model [5- 8 ]. In contrast, in our case, we will adopt an ordering motivated from previous studies performed in the anisotropic Bianchi I model [17]. In such a system, the sign of the triad plays an important role because there are three different directions, rather than one. Then, products of two signs are not necessarily equal to the unity. As a consequence, in that case, one has to take carefully into account the presence of the signs when symmetrizing the constraint. To select a similar ordering in the present isotropic case, we start with the symmetric Hamiltonian constraint constructed in Ref. [17] and identify the three spatial directions. In this manner we arrive to the following constraint operator

$$
\hat{C}:=\left[\frac{\widehat{1}}{V}\right]^{1 / 2}\left(-\frac{6}{\gamma^{2}} \widehat{\Omega}^{2}+8 \pi G \hat{p}_{\phi}^{2}\right)\left[\frac{\hat{1}}{V}\right]^{1 / 2} .
$$

Here, the inverse volume operator is given by

$$
\left[\widehat{\frac{1}{V}}\right]:=\left[\widehat{\frac{1}{\sqrt{|p|}}}\right]^{3}
$$

where

$$
\frac{\widehat{1}}{\sqrt{|p|}}=\frac{3}{4 \pi \gamma l_{\mathrm{Pl}}^{2} \sqrt{\Delta}} \widehat{\operatorname{sign}(p)} \widehat{\sqrt{|p|}}\left(\hat{\mathcal{N}}_{-\bar{\mu}} \widehat{\sqrt{|p|}} \hat{\mathcal{N}}_{\bar{\mu}}-\hat{\mathcal{N}}_{\bar{\mu}} \widehat{\sqrt{|p|}} \hat{\mathcal{N}}_{-\bar{\mu}}\right) .
$$

The inverse volume acts diagonally on the basis states $|v\rangle$ and annihilates the state $|v=0\rangle$. 
On the other hand the operator

$$
\widehat{\Omega}:=\frac{1}{4 i \sqrt{\Delta}}\left[\frac{\widehat{1}}{\sqrt{|p|}}\right]^{-1 / 2} \widehat{\sqrt{|p|}}\left[\left(\hat{\mathcal{N}}_{2 \bar{\mu}}-\hat{\mathcal{N}}_{-2 \bar{\mu}}\right) \widehat{\operatorname{sign}(p)}+\widehat{\operatorname{sign}(p)}\left(\hat{\mathcal{N}}_{2 \bar{\mu}}-\hat{\mathcal{N}}_{-2 \bar{\mu}}\right)\right] \widehat{\sqrt{|p|}}\left[\frac{\widehat{1}}{\sqrt{|p|}}\right]^{-1 / 2}
$$

coincides (in terms of holonomies and triad operators) with the one studied in Ref. [17], denoted there by $\hat{\Theta}_{i}$. The factors involving powers of the triad at the beginning and the end of this expression appear in a very particular manner, in analogy to the Bianchi I model [17]. Nonetheless, this concrete ordering is not relevant inasmuch as physical results do not depend appreciably on it, and one could select any other one. For instance, one could even ignore the quantum effects coming from the inverse of the volume [25], as was done in Ref. [8]. The really relevant features of our ordering, as we will see in the next section, come from the particular treatment of the sign of $p$ that we have considered.

It is straightforward to see that our Hamiltonian constraint operator annihilates the state $|v=0\rangle$ and leaves invariant its orthogonal complement, which we will denote by $\widetilde{\mathcal{H}}_{\text {kin }}^{\text {grav }}$. Hence, when studying the non-trivial solutions of the Hamiltonian constraint, we can restrict ourselves to this latter subspace. Note

that $\widetilde{\mathcal{H}}_{\text {kin }}^{\text {grav }}$ is just the Cauchy completion with the discrete norm of $\widetilde{\mathrm{Cyl}}_{S}$, the linear span of all the $|v\rangle$ states with $v \neq 0$. As in similar situations studied by us in previous works 17, 18, 20, 24], the big bang is then resolved in the sense that the quantum equivalent to the classical singularity (namely, the eigenstate of vanishing physical volume) has been entirely removed from the kinematical Hilbert space.

Once we have removed the kernel of the inverse volume operator, we can introduce the densitized version of $\hat{C}$. Let us remember before that the physical states, which are annihilated by the Hamiltonian constraint, are generally not normalizable in the kinematical Hilbert space. We should seek them in a larger space. A natural home, as far as the gravitational part of the system is concerned, is the algebraic dual $\widetilde{\mathrm{Cyl}}_{S}^{*}$ of the dense set $\widetilde{\mathrm{Cyl}}_{S}$. In this dual space (tensor product any suitable space for the matter degrees of freedom), we can establish a one-to-one relation between any element $(\psi \mid$ annihilated by the (adjoint of the) operator $\hat{C}$ and any other element $\left(\psi^{\prime} \mid=\left(\psi \mid[\widehat{1 / V}]^{1 / 2}\right.\right.$ annihilated by the (adjoint of the) densitized version of the constraint, which is given by

$$
\hat{\mathcal{C}}=-\frac{6}{\gamma^{2}} \widehat{\Omega}^{2}+8 \pi G \hat{p}_{\phi}^{2}
$$

This equivalent form of the Hamiltonian constraint is easier to impose since obviously $\widehat{\Omega}^{2}$ and $\hat{p}_{\phi}^{2}$ are Dirac observables which commute.

Another way to get an easily solvable Hamiltonian constraint is to directly promote the classical densitized constraint $\mathcal{C}:=V C$ to an operator. Nevertheless, as we have commented, such an object does not arise from the standard densitization of the constraint in LQG. For this reason, it seems natural to respect this latter densitization and show that one can construct a bijection between the solutions to the two considered Hamiltonian constraints. We emphasize that this bijection cannot be established in the kinematical Hilbert space, both because the physical volume operator is unbounded, and therefore it is not defined in the whole space, and because solutions do not belong to this space indeed.

\section{CHARACTERIZATION OF THE GRAVITATIONAL CONSTRAINT OPERATOR}

As we have already seen, the operator $\widehat{\Omega}^{2}$, which provides the gravitational part of the Hamiltonian constraint (8), is a Dirac observable. Since the matter part of this Hamiltonian constraint is well known, the study of the properties of $\widehat{\Omega}^{2}$, and in particular its spectral analysis, is the essential step for the resolution of the constraint. In this section, we will carry out a detailed analysis of this operator. We will identify first the superselection sectors which arise in the theory. Afterwards, we will perform the spectral analysis of $\widehat{\Omega}^{2}$ in those sectors and determine its eigenfunctions. Remarkably, we can obtain their explicit expression, what will allow us to study their behavior. On the one hand, we will discuss how these eigenfunctions realize the commented no-boundary description. On the other hand, we will relate them with the eigenfunctions of the geometrodynamical (WDW) counterpart of $\widehat{\Omega}^{2}$ for large $v$, in order to understand the WDW limit of our theory. Both features will give us insights about the existence of a quantum bounce. As a complement, we will also relate $\widehat{\Omega}^{2}$ with the operator $\widehat{\Omega}$. Finally, we will compare our results with those of previous works on the polymeric quantization of the model, to point out the goodness of our prescription. 


\section{A. Superselection Sectors}

In LQC, owing to the discreteness of the volume representation, the gravitational part of the Hamiltonian constraint turns out to be a difference operator instead of a differential one, as happens to be the case in the standard WDW theory. In the particular case of isotropic cosmologies, this operator produces a shift of four units in the label of the basis states $|v\rangle$. As a consequence, only basis states with support in discrete lattices of step four are related under its action. In addition, the Hilbert spaces of states which have support in such lattices turn out to be superselected, inasmuch as they are also preserved under the action of the complete Hamiltonian constraint and the physical observables. Whereas these results are general within isotropic LQC, in this subsection we will see that the lattices associated with our operator $\widehat{\Omega}^{2}$, and the corresponding superselection sectors, are simpler than those obtained in previous studies of the model.

The action of $\widehat{\Omega}^{2}$ on the basis states $|v\rangle$ of $\widetilde{\mathcal{H}}_{\text {kin }}^{\text {grav }}$ takes the form

$$
\widehat{\Omega}^{2}|v\rangle=-f_{+}(v) f_{+}(v+2)|v+4\rangle+\left[f_{+}^{2}(v)+f_{-}^{2}(v)\right]|v\rangle-f_{-}(v) f_{-}(v-2)|v-4\rangle,
$$

where

$$
\begin{aligned}
& f_{ \pm}(v):=\frac{\pi \gamma l_{\mathrm{Pl}}^{2}}{3} g(v \pm 2) s_{ \pm}(v) g(v), \\
& s_{ \pm}(v):=\operatorname{sign}(v \pm 2)+\operatorname{sign}(v)
\end{aligned}
$$

and

$$
g(v):=\left\{\begin{array}{lll}
|| 1+\left.\frac{1}{v}\right|^{\frac{1}{3}}-\left.\left|1-\frac{1}{v}\right|^{\frac{1}{3}}\right|^{-\frac{1}{2}} & \text { if } & v \neq 0 \\
0 & \text { if } \quad v=0 .
\end{array}\right.
$$

Notice that the combination of signs in the functions $f_{ \pm}(v)$, which comes from the factor ordering that we have chosen to symmetrize the constraint operator, is the responsible of a remarkable property, namely, $f_{-}(v) f_{-}(v-2)=0$ if $v \in(0,4]$, while $f_{+}(v) f_{+}(v+2)=0$ for $v \in[-4,0)$. Therefore, the positive and negative semiaxes, $v>0$ and $v<0$, are decoupled under the action of our operator $\widehat{\Omega}^{2}$, as one can see from Eq. (9). In conclusion, this operator relates only basis states $|v\rangle$ with $v$ belonging to one of the semilattices of step four $\mathcal{L}_{\tilde{\varepsilon}}^{ \pm}:=\{v= \pm(\tilde{\varepsilon}+4 n), n \in \mathbb{N}\}$, where $\tilde{\varepsilon} \in(0,4]$. In other words, $\widehat{\Omega}^{2}$ is well defined in any of the Hilbert spaces $\mathcal{H}_{\tilde{\tilde{\varepsilon}}}^{ \pm}$obtained as the closure of the respective domains $\operatorname{Cyl}_{\tilde{\varepsilon}}^{ \pm}:=\operatorname{span}\left\{|v\rangle, v \in \mathcal{L}_{\tilde{\varepsilon}}^{ \pm}\right\}$ with respect to the discrete inner product. Note that the non-separable kinematical Hilbert space $\widetilde{\mathcal{H}}_{\text {kin }}^{\text {grav }}$ can be written as a direct sum of separable subspaces in the form $\widetilde{\mathcal{H}}_{\text {kin }}^{\text {grav }}=\oplus_{\tilde{\varepsilon}}\left(\mathcal{H}_{\tilde{\varepsilon}}^{+} \oplus \mathcal{H}_{\tilde{\varepsilon}}^{-}\right)$.

The Hilbert spaces $\mathcal{H}_{\tilde{\varepsilon}}^{ \pm} \otimes L^{2}(\mathbb{R}, d \phi)$ are preserved by the action of the Hamiltonian constraint (and then, as we will see, of physical observables). Thus, these Hilbert spaces provide superselection sectors in our theory. We can restrict the study to any of them. For concreteness, in the following we will work in $\mathcal{H}_{\tilde{\varepsilon}}^{+} \otimes L^{2}(\mathbb{R}, d \phi)$.

The difference with respect to previous works on the polymeric quantization of this model [5, 8, 10, 11] is that the support of our sectors is contained in a single semiaxis of the real line, while in those works the sectors had contributions both from the positive and the negative semiaxes, which were not decoupled. Later on, we will discuss the advantages of working with our simpler sectors.

\section{B. Spectral Analysis}

Let us begin by showing that the symmetric operator $\widehat{\Omega}^{2}$ (with domain $\mathrm{Cyl}_{\tilde{\varepsilon}}^{+}$) is essentially self-adjoint. Actually, one can calculate that the difference between $\alpha \widehat{\Omega}^{2}$, with $\alpha:=3 /\left(4 \pi \gamma^{2} l_{\mathrm{Pl}}^{2} \hbar\right)$, and the gravitational constraint operator $H_{\text {APS }}^{\prime}$ of Ref. [10], both being defined in the Hilbert space $\mathcal{H}_{\tilde{\varepsilon}}^{+} \oplus \mathcal{H}_{4-\tilde{\varepsilon}}^{-}$for $\tilde{\varepsilon} \neq 4$ (with natural domain $\mathrm{Cyl}_{\tilde{\varepsilon}}^{+} \cup \mathrm{Cyl}_{4-\tilde{\varepsilon}}^{-}$), turns out to be a symmetric trace class operator. We obtain the same conclusion in the particular case $\tilde{\varepsilon}=4$ where, starting with the operator $\alpha \widehat{\Omega}^{2}$ defined in $\mathcal{H}_{4}^{+} \oplus \mathcal{H}_{-4}^{-}$, we have to define its action on $|v=0\rangle$, e.g. by annihilation, since the operator $H_{\mathrm{APS}}^{\prime}$ does not decouple the state $|v=0\rangle$. Taking into account that $H_{\mathrm{APS}}^{\prime}$ was already proven to be essentially self-adjoint [10], a well known theorem by Kato and Rellich [26] ensures that so is $\widehat{\Omega}^{2}$ as well. 
In order to show that its restriction to, e.g., $\mathcal{H}_{\tilde{\varepsilon}}^{+}$is also essentially self-adjoint, we will apply that, if $\widehat{A}$ is a symmetric operator defined in certain Hilbert space $\mathcal{H}$ and $\rho$ is any non-real number, then the operator is essentially self-adjoint if and only if there exists no solution $|\phi\rangle \in \mathcal{H}$ to the so-called deficiency index equation, $\widehat{A}^{\dagger}|\phi\rangle=\rho|\phi\rangle$ [27]. Let us suppose that $\widehat{\Omega}^{2}$ defined in $\mathcal{H}_{\tilde{\varepsilon}}^{+}$were not essentially self-adjoint; this would mean that there exists a non-trivial solution to its deficiency index equation belonging to $\mathcal{H}_{\tilde{\varepsilon}}^{+}$, which in turn would provide a normalizable solution (identically vanishing in $\mathcal{H}_{4-\tilde{\varepsilon}}^{-}$) when the operator is defined in the larger Hilbert space $\mathcal{H}_{\tilde{\varepsilon}}^{+} \oplus \mathcal{H}_{4-\tilde{\varepsilon}}^{-}$. We would then reach a contradiction because we already know that the operator is essentially self-adjoint in this larger space. Therefore, $\widehat{\Omega}^{2}$ has to be essentially self-adjoint in $\mathcal{H}_{\tilde{\varepsilon}}^{+}$, as we wanted to prove.

On the other hand, it was shown in Ref. [10] that the essential and the absolutely continuous spectra 27] of the operator $H_{\mathrm{APS}}^{\prime}$ are both $[0, \infty)$. Once again, Kato's perturbation theory [26] allows us to extend these results to our operator $\widehat{\Omega}^{2}$ defined in $\mathcal{H}_{\tilde{\varepsilon}}^{+} \oplus \mathcal{H}_{4-\tilde{\varepsilon}}^{-}$, since (up to a global factor) it differs from $H_{\mathrm{APS}}^{\prime}$ in a symmetric trace class operator. In addition, taking into account the symmetry of $\widehat{\Omega}^{2}$ under a flip of sign in $v\left[f_{ \pm}(-v)=-f_{\mp}(v)\right]$ and assuming the independence of the spectrum in the label $\tilde{\varepsilon}$, we conclude that the operator $\widehat{\Omega}^{2}$ defined in $\mathcal{H}_{\tilde{\varepsilon}}^{+}$is a positive (essentially) self-adjoint operator whose essential and absolutely continuous spectra are $[0, \infty)$ as well. Besides, as we will see in Subsec. IVD, the (generalized) eigenfunctions of $\widehat{\Omega}^{2}$ converge for large $v$ to eigenfunctions of the WDW counterpart of the operator. This fact, together with the continuity of the spectrum in geometrodynamics, suffices to conclude that the discrete and singular spectra are empty [28].

\section{Generalized Eigenfunctions}

Let $\left|e_{\lambda}^{\tilde{\varepsilon}}\right\rangle=\sum_{v \in \mathcal{L}_{\tilde{\varepsilon}}^{+}} e_{\lambda}^{\tilde{\varepsilon}}(v)|v\rangle$ denote a generalized eigenstate of $\widehat{\Omega}^{2}$ corresponding to the generalized eigenvalue $\lambda \in[0, \infty)$. For all $n \in \mathbb{N}^{+}$, each coefficient $e_{\lambda}^{\tilde{\varepsilon}}(\tilde{\varepsilon}+4 n)$ of this generalized eigenfunction turns out to be determined by the single initial datum $e_{\lambda}^{\tilde{\varepsilon}}(\tilde{\varepsilon})$ in the following manner

$$
e_{\lambda}^{\tilde{\varepsilon}}(\tilde{\varepsilon}+4 n)=\left[\mathcal{S}_{\tilde{\varepsilon}}(0,2 n)+\frac{F(\tilde{\varepsilon})}{G_{\lambda}(\tilde{\varepsilon}-2)} \mathcal{S}_{\tilde{\varepsilon}}(1,2 n)\right] e_{\lambda}^{\tilde{\varepsilon}}(\tilde{\varepsilon}),
$$

where

$$
F(v):=\frac{f_{-}(v)}{f_{+}(v)}, \quad G_{\lambda}(v):=-\frac{i \sqrt{\lambda}}{f_{+}(v)},
$$

and

$$
\mathcal{S}_{\tilde{\varepsilon}}(a, b):=\sum_{O(a \rightarrow b)}\left[\prod_{\left\{r_{p}\right\}} F\left(\tilde{\varepsilon}+2 r_{p}+2\right) \prod_{\left\{s_{q}\right\}} G_{\lambda}\left(\tilde{\varepsilon}+2 s_{q}\right)\right] .
$$

Here, $O(a \rightarrow b)$ denotes the set of all possible ways to move from $a$ to $b$ by jumps of one or two unit steps. For each element in $O(a \rightarrow b),\left\{r_{p}\right\}$ is the subset of integers followed by a jump of two units, whereas $\left\{s_{q}\right\}$ is the subset of integers followed by a jump of only one unit. Note that $F(\tilde{\varepsilon})=0$ for all $\tilde{\varepsilon} \leq 2$, so that in these cases the second term in Eq. (13) does not contribute.

As we stated above, the spectrum of $\widehat{\Omega}^{2}$ is positive and absolutely continuous. In terms of a basis of generalized eigenstates $\left|e_{\lambda}^{\tilde{\varepsilon}}\right\rangle$, the spectral resolution of the identity $\mathbb{I}$ in the kinematical Hilbert space $\mathcal{H}_{\tilde{\varepsilon}}^{+}$ is given by

$$
\mathbb{I}=\int_{\mathbb{R}^{+}} d \lambda\left|e_{\lambda}^{\tilde{\varepsilon}}\right\rangle\left\langle e_{\lambda}^{\tilde{\varepsilon}}\right|
$$

Note that the integral runs just over the positive semiaxis and the spectrum is non-degenerate. The eigenfunctions satisfy the $\delta$-normalization condition $\left\langle e_{\lambda}^{\tilde{\varepsilon}} \mid e_{\lambda^{\prime}}^{\tilde{\varepsilon}}\right\rangle=\delta\left(\lambda-\lambda^{\prime}\right)$. This condition fixes the norm of $e_{\lambda}^{\tilde{\varepsilon}}(\tilde{\varepsilon})$ in Eq. (13). The only remaining freedom is then the phase of this initial datum. We finally fix this phase by taking $e_{\lambda}^{\tilde{\varepsilon}}(\tilde{\varepsilon})$ positive. The generalized eigenfunctions that form the basis are then real, a consequence of the fact that the difference operator $\widehat{\Omega}^{2}$ has real coefficients. 
It is worth emphasizing that we have been able to solve the general eigenvalue equation of our gravitational constraint operator $\widehat{\Omega}^{2}$, determining explicitly the form of its generalized eigenfunctions. This contrasts with the level of resolution achieved in Ref. [7], where the generalized eigenfunctions of the corresponding gravitational constraint operator were given in an iterative form and generated numerically. In this respect, we are in an optimal situation to progress in the comprehension of our system since we can now study its behavior analytically. In comparison with the exactly solvable model of Ref. [8], here we do not need to introduce simplifications in the system nor restrict the study to a particular sector of superselection, but our results are completely general. Remember that the construction of Ref. [8] was applied to a simplified version of the model and only in a specific sector whose support is centered symmetrically around $v=0$.

\section{Wheeler-DeWitt Limit}

Another important issue that we want to investigate is the behavior of the quantum physical states (which we will determine in Sec. VD in the region of large volume. In particular, we want to discuss whether one recovers in that region the standard quantization performed in geometrodynamics, namely the WDW theory, whose predictions (for expectation values) in turn agree on semiclassical states with the classical ones obtained from General Relativity. To carry out such analysis, we will only need to know how the eigenfunctions of the gravitational constraint operator $\widehat{\Omega}^{2}$ behave in the large $v$ limit, since the other operator involved in the densitized Hamiltonian constraint, $\hat{p}_{\phi}^{2}$, has already been quantized in terms of the standard "Schroedinger-like" representation. In this subsection, we will obtain the eigenfunctions of the WDW analog of the operator $\widehat{\Omega}^{2}$ and relate them with the eigenfunctions $e_{\lambda}^{\tilde{\varepsilon}}(v)$ for large $v$.

As above for LQC, in the WDW quantization we work in the triad representation. The gravitational part of the kinematical Hilbert space of the WDW quantization can then be chosen as the space of square integrable functions of $v$ with respect to the Lebesgue measure. The operator $\hat{p}$ acts by multiplication by the factor $p=\operatorname{sign}(v)\left(2 \pi \gamma l_{\mathrm{Pl}}^{2} \sqrt{\Delta}|v|\right)^{2 / 3}$, just as in the loop quantization, and the connection is represented by the derivative operator $\hat{c}=i 2\left(2 \pi \gamma l_{\mathrm{Pl}}^{2} / \Delta\right)^{1 / 3}|v|^{1 / 6} \partial_{v}|v|^{1 / 6}$, so that $[\hat{c}, \hat{p}]=i \hbar \widehat{\{c, p\}}$.

Let us denote by $\underline{\widehat{\Omega}}^{2}$ the operator counterpart of the classical quantity $(c p)^{2}$ in the WDW theory [defined in the Schwartz space $\mathcal{S}(\mathbb{R})$ ]. Since we want to compare its features with those of $\widehat{\Omega}^{2}$, we choose for it the analog factor ordering, which gives rise to a symmetric operator that is well defined in the distributional sense:

$$
\underline{\Omega}^{2}=-\beta^{2} \sqrt{|v|}\left[\operatorname{sign}(v) \partial_{v}+\partial_{v} \operatorname{sign}(v)\right]|v|\left[\operatorname{sign}(v) \partial_{v}+\partial_{v} \operatorname{sign}(v)\right] \sqrt{|v|}=-\beta^{2}\left[1+4 v \partial_{v}+4\left(v \partial_{v}\right)^{2}\right],
$$

where $\beta:=4 \pi \gamma l_{\mathrm{Pl}}^{2}$. Note that we have simplified the expression of this operator by disregarding the non-contributing term $|v| \delta(v)$ in the second equality.

Owing to well known properties of the operator $-i v \partial_{v}$, we can ensure that $\underline{\widehat{\Omega}}^{2}$ is not only essentially self-adjoint in $L^{2}(\mathbb{R}, d v)$, but also in each of the subspaces $L^{2}\left(\mathbb{R}^{ \pm}, d v\right)$. Hence, its action on the positive semiaxis $v>0$ and the negative one $v<0$ are decoupled, similarly to what happens with its analog $\widehat{\Omega}^{2}$ in LQC, and we can restrict the study to $L^{2}\left(\mathbb{R}^{+}, d v\right)$. Furthermore, in this latter Hilbert space, the spectrum of $\underline{\Omega}^{2}$ is positive and absolutely continuous. Its generalized eigenfunctions, corresponding to any generalized eigenvalue $\lambda \in[0, \infty)$, can be labeled by $\sigma:= \pm \sqrt{\lambda} \in \mathbb{R}$ and are given by

$$
\underline{e}_{\sigma}(v)=\frac{1}{\sqrt{2 \pi \beta|v|}} \exp \left(-i \sigma \frac{\ln |v|}{\beta}\right) .
$$

They provide a basis for $L^{2}\left(\mathbb{R}^{+}, d v\right)$, normalized so that $\left\langle\underline{e}_{\sigma} \mid \underline{e}_{\sigma^{\prime}}\right\rangle=\delta\left(\sigma-\sigma^{\prime}\right.$ ) (with $\delta$ being the Dirac delta on the real line).

We see that the spectrum of $\widehat{\widehat{\Omega}}^{2}$ has two-fold degeneracy, while we have shown that the spectrum of $\widehat{\Omega}^{2}$ is non-degenerate. Therefore, any loop eigenfunction converges in the large $v$ limit to a linear combination of the two corresponding WDW eigenfunctions (actually, one can rigorously prove that the limit of the loop eigenfunctions indeed exists [18]). Moreover, since the loop eigenfunctions are real, both WDW components must contribute with equal amplitude in that linear combination. Namely, the WDW limit 
has the form

$$
e_{\lambda}^{\tilde{\varepsilon}}(v) \rightarrow r\left\{\exp \left[i \phi_{\tilde{\varepsilon}}(\sigma)\right] \underline{e}_{\sigma}(v)+\exp \left[-i \phi_{\tilde{\varepsilon}}(\sigma)\right] \underline{e}_{-\sigma}(v)\right\}
$$

where $r$ is certain real number. In turn, one can check numerically that the phase shift $\phi_{\tilde{\varepsilon}}(\sigma)$ has the following behavior

$$
\phi_{\tilde{\varepsilon}}(\sigma)=T(|\sigma|)+c_{\tilde{\varepsilon}}+R_{\tilde{\varepsilon}}(|\sigma|) \text {. }
$$

where $T$ is a function of $|\sigma|$ only, $c_{\tilde{\varepsilon}}$ is a constant which depends on $\tilde{\varepsilon}$, and $\lim _{\sigma \rightarrow \infty} R_{\tilde{\varepsilon}}(|\sigma|)=0[18$. So, whereas the eigenfunctions of our operator are determined by a single piece of initial data, they behave as eigenfunctions of a second order differential operator in the large $v$ limit, therefore picking up a particular linear combination of the solutions to the eigenvalue problem of that differential operator. This nice feature of the polymeric quantization is the main responsible of the quantum bounce picture, together with the no-boundary description realized with our superselection sectors, as we will discuss in Sec. VI We postpone also to that section the comparison between the WDW limit of our theory and that of previous quantizations of the model.

\section{E. Operator $\widehat{\Omega}$}

Once we have characterized the gravitational constraint operator $\widehat{\Omega}^{2}$, let us relate it with $\widehat{\Omega}$ to point out some interesting features which are due to the polymeric quantization and that do not appear in the analog WDW theory. We recall that (up to a multiplicative constant factor arising from a change in the basic commutators) $\widehat{\Omega}$ coincides in fact with the operator $\widehat{\Theta}_{i}$ extensively studied in Ref. [17]. We now summarize its properties. Like $\widehat{\Omega}^{2}$, it is a difference operator, but with a constant step of two units in $v$ instead of four. Its action is

$$
\widehat{\Omega}|v\rangle=-i\left[f_{+}(v)|v+2\rangle-f_{-}(v)|v-2\rangle\right],
$$

with $f_{ \pm}(v)$ defined in Eq. (10). Taking into account that $f_{-}(v)=0$ if $v \in(0,2]$ and $f_{+}(v)=0$ when $v \in[-2,0)$, we see that this operator does not mix states $|v\rangle$ with $v$ belonging to different semilattices of the form ${ }^{(2)} \mathcal{L}_{\varepsilon}^{ \pm}:=\{v= \pm(\varepsilon+2 n), n \in \mathbb{N}\}$, where $\varepsilon \in(0,2]$. In particular, our operator is well defined (with a natural choice of domain) in the Hilbert space ${ }^{(2)} \mathcal{H}_{\varepsilon}^{+}:=\mathcal{H}_{\tilde{\varepsilon}=\varepsilon}^{+} \oplus \mathcal{H}_{\tilde{\varepsilon}=\varepsilon+2}^{+}$. Furthermore, from the properties of $\widehat{\Omega}^{2}$, we infer that $\widehat{\Omega}$ defined in ${ }^{(2)} \mathcal{H}_{\varepsilon}^{+}$is an essentially self-adjoint operator whose spectrum is absolutely continuous, non-degenerate, and equal to the real line. In turn, its generalized eigenstates $\left|e_{\sigma}^{\varepsilon}\right\rangle$, with support in ${ }^{(2)} \mathcal{L}_{\tilde{\varepsilon}}^{+}$and corresponding to the generalized eigenvalue $\sigma:= \pm \sqrt{\lambda} \in \mathbb{R}$, are formed by the direct sum of two generalized eigenstates of the squared operator $\widehat{\Omega}^{2}$ for the eigenvalue $\lambda$, one with support in the semilattice of step four $\mathcal{L}_{\tilde{\varepsilon}=\varepsilon}^{+}$and the other supported in the semilattice $\mathcal{L}_{\tilde{\varepsilon}=\varepsilon+2}^{+}$. Explicitly one can see that $\left|e_{\sigma}^{\varepsilon}\right\rangle=\sqrt{|\sigma|}\left[\left|e_{\lambda}^{\varepsilon}\right\rangle \oplus i \operatorname{sign}(-\sigma)\left|e_{\lambda}^{\varepsilon+2}\right\rangle\right]$ for $\sigma \neq 0$, with $\left\langle e_{\sigma}^{\varepsilon} \mid e_{\sigma^{\prime}}^{\varepsilon}\right\rangle=\delta\left(\sigma-\sigma^{\prime}\right)$ (like in the WDW case). For $\sigma=0$, we define $\left|e_{\sigma=0}^{\varepsilon}\right\rangle=\left|e_{\lambda=0}^{\varepsilon}\right\rangle[17]$.

Let us comment that, whereas the eigenfunctions of $\widehat{\Omega}^{2}$ have a well defined continuum limit for large $v$, those of $\widehat{\Omega}$ do not possess such a limit, since they are formed by two components, each of them admitting a WDW limit, but which are shifted by a phase equal to $\pm \pi / 2$ (owing to the factor $\pm i$ in the linear combination). As a consequence, when $v$ varies in ${ }^{(2)} \mathcal{L}_{\tilde{\varepsilon}}^{+}$, the eigenfunctions $e_{\sigma}^{\varepsilon}(v)$ oscillate rapidly. This behavior is not present in the standard WDW theory, where the eigenfunctions of $\underline{\Omega}$ are continuous in $v$ for $v>0$ [they coincide with those of $\underline{\Omega}^{2}$ given in Eq. (18)] .

\section{PHYSICAL HILBERT SPACE}

We can now complete the quantization by obtaining the physical Hilbert space and providing a complete set of observables.

The matter term present in the densitized Hamiltonian constraint has been treated in a standard nonpolymeric way. The (essentially) self-adjoint operator $\hat{p}_{\phi}^{2}$ is positive with a two-fold degenerate spectrum. Its generalized eigenvalues are labeled by $\omega^{2}$, with $\omega \in \mathbb{R}$. Let us call $\mathcal{U}$ the dense domain of definition of $\widehat{\mathcal{C}}$, invariant under its action, from which one obtains the self-adjoint extension of this constraint operator 
$\left(\mathcal{U}\right.$ is the tensor product of, e.g., $\mathrm{Cyl}_{\tilde{\varepsilon}}^{+}$and a suitable domain for $\left.\hat{p}_{\phi}^{2}\right)$. Starting from this invariant domain, we can apply the group averaging method to find the physical Hilbert space $\mathcal{H}_{\tilde{\varepsilon}}^{\text {Phy }}[29$, 30]. The resulting physical states have the form

$$
\Psi(v, \phi)=\int_{0}^{\infty} \frac{d \lambda}{\omega(\lambda)} e_{\lambda}^{\tilde{\varepsilon}}(v)\left\{\psi_{+}(\lambda) \exp [i \omega(\lambda) \phi]+\psi_{-}(\lambda) \exp [-i \omega(\lambda) \phi]\right\},
$$

where $\psi_{+}(\lambda)$ and $\psi_{-}(\lambda)$ belong to the physical Hilbert space $\mathcal{H}_{\tilde{\varepsilon}}^{\text {Phy }}=L^{2}\left(\mathbb{R}^{+}, \omega^{-1}(\lambda) d \lambda\right)$ and $\omega(\lambda)=\sqrt{\alpha \lambda}$ [with $\left.\alpha=3 /\left(4 \pi \gamma^{2} l_{\mathrm{Pl}}^{2} \hbar\right)\right]$. Regarding $\phi$ as the internal time, we see that the solutions can be decomposed in positive $(+)$ and negative $(-)$ frequency components, $\Psi_{ \pm}(v, \phi)$, which are determined by the initial data $\Psi_{ \pm}\left(v, \phi_{0}\right)$ through the unitary evolution $\Psi_{ \pm}(v, \phi)=U_{ \pm}\left(\phi-\phi_{0}\right) \Psi_{ \pm}\left(v, \phi_{0}\right)$, where

$$
U_{ \pm}\left(\phi-\phi_{0}\right)=\exp \left[ \pm i \sqrt{\alpha \widehat{\Omega}^{2}}\left(\phi-\phi_{0}\right)\right] .
$$

A complete set of observables that allows us to interpret the system in an evolution picture is given by the constant of motion $\hat{p}_{\phi}$ and the relational observable $\left.\hat{v}\right|_{\phi_{0}}$ (or $\widehat{|v|}_{\phi_{0}}$ if we do not restrict to $v>0$, see Ref. [7]). The latter measures the value of the volume when the time takes the value $\phi_{0}$. These Dirac observables preserve the positive and negative frequency sectors, so that, apart from the superselection already discussed, there exists further superselection with respect to the frequency. We can hence restrict the study, for instance, to the positive frequency sector.

\section{DISCUSSION AND COMPARISON WITH PREVIOUS WORK}

In this section, we discuss the consequences of the quantization presented here as well as the main similarities and differences with respect to previous works on isotropic LQC, especially Ref. [7], where the physical Hilbert space was originally determined and the quantum bounce of states that are semiclassical at late times was first studied satisfactorily.

Let us remember that the analog of the operator $\widehat{\Omega}^{2}$ in Ref. 7], denoted by $\Theta$, is also (essentially) self-adjoint and positive. Furthermore, the quantum model constructed with $\Theta$ has superselection sectors supported in the lattices of step four $\mathcal{L}_{ \pm|\epsilon|}:=\{v= \pm|\epsilon|+4 n, n \in \mathbb{Z}\}$, where $|\epsilon| \in[0,2]$. As we see, these lattices extend over the real line. In these superselection sectors, the spectrum of $\Theta$ is absolutely continuous and two-fold degenerate. Associated with this operator, a particular $(\delta$-)orthonormal basis of generalized eigenfunctions was chosen in Ref. [7]. Their elements were denoted by $e_{k}(v)$ with $k \in \mathbb{R}$, so that $e_{|k|}(v)$ and $e_{-|k|}(v)$ have the same eigenvalue. This basis is defined in such a way that $e_{-|k|}(v)$ tends to $\underline{e}_{-|k|}(v)$ for large positive $v$, where $\underline{e}_{-|k|}(v)$ is the generalized eigenfunction of the WDW operator analog of $\Theta$ that provides an expanding wave. Similarly, we call $\underline{e}_{|k|}(v)$ the WDW eigenfunction corresponding to a contracting wave. The asymptotic behavior of $e_{-|k|}(v)$ turns out to be given then by

$$
e_{-|k|}(v) \stackrel{v \gg 1}{\longrightarrow} \underline{e}_{-|k|}(v), \quad e_{-|k|}(v) \stackrel{v \ll-1}{\longrightarrow} A \underline{e}_{-|k|}(v)+B \underline{e}_{|k|}(v) .
$$

Numerical analysis has shown that, for large $|k|, A$ and $B$ satisfy $|A|^{2}-|B|^{2}=1$ and $|A| \sim|B| \gg 1$. As a result, the eigenfunctions $e_{-|k|}(v)$ suffer an amplification in the negative semiaxis. In addition, this amplification is stronger as $|k|$ increases.

On the other hand, since the parity transformation $v \rightarrow-v$ is a (large) gauge symmetry of the theory, in Ref. 7] the analysis was reduced to the symmetric sector. Then, in general, it was necessary to join two different lattices so that the support of the states was symmetrically distributed around $v=0$. Thanks to the introduction of that symmetry, the analysis could be restricted to the positive $v$-semiaxis.

Besides, in that work, the study was limited to the most interesting physical states: those which are semiclassical at late times. These states are provided by Gaussian profiles peaked at a large momentum of the scalar field or, equivalently, at a large negative $k$. Thus, in such regime the contribution of $e_{|k|}(v)$ to the physical solutions is negligible. In this situation the form of the generalized eigenfunctions $e_{|k|}(v)$ is irrelevant, and it was not necessary to calculate them.

Once the parity symmetry is introduced and physical states are restricted in practice to the region of large negative $k$ 's, one attains in Ref. [7] a similar scenario to ours, in the sense that, for large positive $v$, the symmetric eigenfunctions that contribute significantly have a WDW limit in each lattice $\mathcal{L}_{ \pm|\epsilon|}$ which is approximately of standing-wave type. This is just a consequence of Eq. (24) and the commented 
properties of the coefficients $A$ and $B$, together with the implementation of the parity transformation. However, it is worth emphasizing that, while this standing-wave behavior is just an approximation valid for $k \ll-1$ in the case of Ref. 7], in our model this behavior is reached exactly and for all the eigenvalues of the scalar field momentum in the WDW limit.

As for the procedure of Ref. 7] to restrict to states in the parity symmetric sector, which leads to the mentioned union of two different lattices for generic $|\epsilon|$ (namely, $|\epsilon| \neq 0,2)$, this has some consequences which deserve special comment. Even when restricting the analysis to the sector $k \ll-1$, the WDW limit of the eigenfunctions carries a constant phase shift which depends on $\epsilon$. Actually, one can see numerically that the relative phase of the coefficients $A$ and $B$ presents the same kind of dependence found for our model in Eq. (20) (with $\tilde{\varepsilon}$ and $|\sigma|$ now replaced with $\epsilon$ and $|k|$ ). Therefore, even in the region of interest $k \ll-1$, two different lattices possess different WDW limits and then their union does not admit a global limit. Remarkably, for the semiclassical states considered in Ref. [7], which are peaked for $v \gg 1$ around two classical trajectories that do not overlap (one of an expanding universe and the other of a contracting one), the difference in the WDW limit of the two lattices is just a global phase for each of the two mentioned branches, which neither affects the norm of the state nor the expectation values of the observables. Nonetheless, this property of the WDW limit is not valid for more general states.

The model studied in Ref. 7] was later simplified in Ref. [8], mainly by disregarding the quantum corrections associated with the inverse volume operator. This simplification permitted to obtain an exactly solvable model in which the quantum bounce was shown in fact to be generic, although this result was attained, however, only for a specific superselection sector, namely, the one containing the state $|v=0\rangle$.

In comparison with Refs. 7, [8], a distinction of our proposal is that all the superselection sectors have a support contained in a single semiaxis. This allows us to restrict the study to e.g. $v \in \mathbb{R}^{+}$in a natural way, without the need to appeal to a symmetrization process such as the one described above. In any case, note that the sectors of our model are perfectly compatible with the imposition of parity symmetry, which can be directly implemented by taking the direct sum of two sectors with support in the union of two semilattices, $\mathcal{L}_{\tilde{\varepsilon}}^{+} \cup \mathcal{L}_{\tilde{\varepsilon}}^{-}$. Owing to the simplicity of our sectors, the spectrum of $\widehat{\Omega}^{2}$ is non-degenerate, what facilitates the exact and explicit calculation of the whole basis of generalized eigenfunctions [see Eq. (13)]. An analytical and numerical advantage of this non-degeneracy is that, to fix each eigenfunction, we only need to impose the positiveness of the initial datum $e_{\lambda}^{\tilde{\varepsilon}}(\tilde{\varepsilon})$, because its norm is completely determined by the normalization condition. As we have explained above, the WDW limit of these eigenfunctions takes the form (19). It is a combination of two WDW eigenfunctions which can be interpreted as contracting and expanding components (in $v$ ), or equivalently as incoming and outgoing components. They contribute with equal amplitude since the eigenfunctions are real, and in this sense the limit is exactly a standing wave. On the other hand, our eigenfunctions have support in a semiaxis which does not contain the potential singularity. This behavior does not arise from the imposition of any particular condition, like e.g. a boundary condition, but it is a natural feature of our model, explainable only by the functional properties of our gravitational constraint operator. From this perspective, we consider that our model provides an intrinsic no-boundary description. This implies that the outgoing component must evolve to an incoming component and vice versa, since the flux cannot scape across $v=0$. Therefore, the expanding and contracting components must represent the two branches of a bouncing universe. Unlike in Ref. 7], where only a certain regime is considered, this result is independent of the spectral profile of interest. Furthermore, our analysis is valid for all choices of superselection sector (i.e., for all values of $\tilde{\varepsilon}$ ), in contrast with the discussion carried out in Ref. [8]. In short, we obtain a completely generic quantum bounce. Obviously, the commented expanding and contracting components will be peaked (for large $v$ ) around well differentiated trajectories only for certain types of states.

Let us conclude by remarking that the kind of no-boundary description that we have reached, which plays a key role in the arguments leading to the picture of a quantum bounce, is a characteristic of our model that is not shared by any of the previous works on flat isotropic LQC [5, 8, 10, 11].

\section{CONCLUSION}

We have presented an alternative quantization in LQC of a flat, homogeneous, and isotropic universe in the presence of a massless scalar (homogeneous) field with the aim of improving our understanding of the theory. More explicitly, by a new choice of factor ordering, motivated by studies of anisotropic cosmologies, we have symmetrized the Hamiltonian constraint in a way which results specially appropriate to investigate some issues which had not been taken into account with sufficient care in previous analyses, 
such us the precise relation between the Hamiltonian constraint and its densitized version, the possibility to attain superselection sectors which, being as simple as possible, posses optimal properties, and a clear comprehension of the WDW limit of the theory.

Our Hamiltonian constraint operator presents two nice features: under its action, the zero-volume state decouples, and states with different orientation of the triad are not mixed. Owing to the former of these properties, in our model the big bang singularity is kinematically resolved inasmuch as its quantum analog, namely the zero-volume state, is removed from the kinematical Hilbert space. This fact allows us to establish a bijection between the non-trivial solutions of the Hamiltonian constraint (with densitization equal to that used in LQG) and those of its densitized version (with respect to the volume). This bijection is not established in the kinematical Hilbert space, but in a larger space that provides a natural home for the solutions. For the densitized version of the constraint, the identification of some Dirac observables is straightforward. In summary, our analysis shows in a rigorous way how one can start with the density weight for the Hamiltonian constraint which arises naturally in LQG and make the passage to a simpler constraint with different densitization.

The second feature commented above, namely the decoupling between triads with different orientation, has also important consequences. Thanks to it, our Hamiltonian constraint superselects sectors with support in a single semiaxis of the real line, instead of the whole real line. The simplicity of our sectors in turn simplifies considerably the construction of the physical Hilbert space in comparison with previous works [5 -8, 10, 11]. In those references the superselection sectors have support in lattices of the real line. The study can be restricted to the positive semiaxis $v>0$ by demanding parity symmetry, but in the generic case this is made at the cost of replacing the original individual lattices by the union of two lattices that are transformed one into each other under parity. In contrast, in our case the functional properties of the gravitational constraint operator restrict the study to $v>0$ directly. As a consequence, the generalized eigenfunctions of this constraint operator, which are the elements that account for the quantum information about the geometry, provide a no-boundary description, arising in a single section of minimum non-zero volume $v=\varepsilon$ without the need to introduce any specific (boundary or large gauge) condition in order to affect the behavior in the vicinity of the origin. Another related consequence is that the spectrum of the gravitational constraint operator is non-degenerate, whereas the spectrum of the analog WDW operator is two-fold degenerate. Taking into account that the eigenfunctions of the loop operator can be chosen to be real, they must converge in the WDW limit (large $v$ limit) to a standing wave composed of two WDW eigenfunctions equally contributing, one outgoing and the other incoming. In turn, the no-boundary behavior implies that neither the incoming flux nor the outgoing one can "scape" to the region $v<0$. Thus, the only possibility is a bounce in which the incoming component becomes outgoing and vice versa. This shows the occurrence of a quantum bounce in a generic manner. Our conclusions do not only confirm the results obtained in previous works [7, 8], in particular concerning the robustness of the bounce, but also reinforce them inasmuch as the discussed scenario is completely general. We have neither focused the study on a concrete class of physical states, unlike in Ref. [7], nor simplified and particularized it to a specific superselection sector, unlike in Ref. [8]. Finally, we note that the commented bounce scenario does not imply that the outgoing and ingoing components peak at non-overlapping classical trajectories, so that such a semiclassical behavior will be reached only for certain physical states (e.g. those considered in Ref. [7]).

In conclusion, the simplicity of our quantum model has allowed us to solve it explicitly, gaining the physical intuition necessary to develop our understanding of LQC without resorting to numerical analyses. In this way, we have improved the control over the WDW limit and analyzed in depth the fundamental reasons behind the occurrence of a quantum bounce.

\section{ACKNOWLEDGMENTS}

This work was supported by the Spanish MICINN Project FIS2008-06078-C03-03 and the ConsoliderIngenio 2010 Program CPAN (CSD2007-00042). The authors are grateful to D. Brizuela, L.J. Garay, T. Pawłowski, R. Rodríguez-Oliveros, and J.M. Velhinho for discussions. M.M.-B. acknowledges financial aid by the I3P Program of CSIC and the European Social Fund under the grant I3P-BPD2006. She thanks A.E.I. for warm hospitality during part of the period of development of this work. J.O. acknowledges 
CSIC by financial support under the grant JAEPre_08_00791.

[1] T. Thiemann, Modern Canonical Quantum General Relativity (Cambridge University Press, Cambridge, England, 2007).

[2] C. Rovelli, Quantum Gravity (Cambridge University Press, Cambridge, England, 2004).

[3] A. Ashtekar and J. Lewandowski, Classical Quantum Gravity 21, R53 (2004).

[4] M. Bojowald, Living Rev. Rel. 11, 4 (2008).

[5] A. Ashtekar, M. Bojowald, and J. Lewandowski, Adv. Theor. Math. Phys. 7, 233 (2003).

[6] A. Ashtekar, T. Pawłowski, and P. Singh, Phys. Rev. Lett. 96, 141301 (2006); Phys. Rev. D 73, 124038 (2006).

[7] A. Ashtekar, T. Pawłowski, and P. Singh, Phys. Rev. D 74, 084003 (2006).

[8] A. Ashtekar, A. Corichi, and P. Singh, Phys. Rev. D 77, 024046 (2008).

[9] A. Corichi and P. Singh, Phys. Rev. Lett. 100, 161302 (2008); Phys. Rev. D 80, 044024 (2009).

[10] W. Kamiński and J. Lewandowski, Classical Quantum Gravity 25, 035001 (2008).

[11] W. Kamiński, J. Lewandowski, and T. Pawłowski. Classical Quantum Gravity 26, 035012 (2009).

[12] A. Ashtekar, T. Pawłowski, P. Singh, and K. Vandersloot, Phys. Rev. D 75, 024035 (2007).

[13] K. Vandersloot, Phys. Rev. D 75, 023523 (2007).

[14] L. Szulc, W. Kamiński, and J. Lewandowski, Classical Quantum Gravity 24, 2621 (2007).

[15] E. Bentivegna and T. Pawłowski, Phys. Rev. D 77, 124025 (2008).

[16] D.W. Chiou, Phys. Rev. D 75, 024029 (2007).

[17] M. Martín-Benito, G.A. Mena Marugán, and T. Pawłowski, Phys. Rev. D 78, 064008 (2008).

[18] M. Martín-Benito, G.A. Mena Marugán, and T. Pawłowski, Phys. Rev. D 80, 084038 (2009).

[19] A. Ashtekar and E. Wilson-Ewing, Phys. Rev. D 79, 083535 (2009).

[20] M. Martín-Benito, L.J. Garay, and G.A. Mena Marugán, Phys. Rev. D 78, 064008 (2008); G.A. Mena Marugán and M. Martín-Benito, Int. J. Mod. Phys. A 24, 2820 (2009).

[21] M. Bojowald, Classical Quantum Gravity 20, 2595 (2003).

[22] Here $\tau_{i}$ are elements of the su(2) algebra, proportional to the Pauli matrices, and satisfy $\tau_{i} \tau_{j}=\frac{1}{2} \varepsilon_{i j k} \tau^{k}-\frac{1}{4} \delta_{i j}$.

[23] J.M. Velhinho, Classical Quantum Gravity 24, 3745 (2007).

[24] G.A. Mena Marugán, AIP Conf. Proceedings 1130, 89 (2009).

[25] This would also eliminate possible dependences on the choice of fiducial cell in the effective theory at subleading orders. See e.g. Y. Ding, Y. Ma, and J. Yang, Phys. Rev. Lett. 102, 051301 (2009).

[26] T. Kato, Perturbation Theory for Linear Operators (Springer-Verlag, Berlin, 1980).

[27] See e.g. A. Galindo and P. Pascual, Quantum Mechanics I (Springer-Verlag, Berlin, 1990); M. Reed and B. Simon, Methods of Modern Mathematical Physics I: Functional Analysis (Academic Press, San Diego, 1980).

[28] W. Kamiński (unpublished).

[29] D. Marolf, arXiv:gr-qc/9508015; Classical Quantum Gravity 12, 1199 (1995); 12, 1441 (1995); 12, 2469, (1995).

[30] A. Ashtekar, J. Lewandowski, D. Marolf, J. Mourão, and T. Thiemann, J. Math. Phys. 36, 6456 (1995). 